FTUV/98/60

IFIC $/ 98 / 61$

July 1998

\title{
Cascade decays of triplet Higgs bosons at LEP2.
}

\author{
A.G. Akeroyd \\ Departamento de Física Teórica, IFIC/CSIC, \\ Universidad de Valencia, Burjassot 46100, \\ Valencia, Spain
}

\begin{abstract}
We study the Georgi-Machacek two triplet, one doublet model in the context of LEP2, and show that cascade decays of Higgs bosons to lighter Higgs bosons and a virtual vector boson may play a major role. Such decays would allow the Higgs bosons of this model to escape current searches, and in particular are of great importance for the members of the five-plet which will always decay to the three-plet giving rise to cascade signatures.
\end{abstract}

\footnotetext{
1akeroyd@flamenco.ific.uv.es
} 


\section{Introduction}

It is well known that the Standard Model (SM) [1] requires a Higgs sector [2] in order to break the electroweak symmetry and allow massive fermions and gauge bosons. Complex Higgs doublets (and singlets) are the most natural way of achieving this because they predict $\rho=M_{W}^{2} /\left(M_{Z}^{2} \cos ^{2} \theta_{W}\right)=1$ at tree-level [3]. With the present experimental value measured to be $1.0012 \pm 0.0013 \pm 0.0018$ [4], such a tree-level result is preferable. The minimal SM possesses one complex doublet which after symmetry breaking predicts one physical neutral scalar $\left(\phi^{0}\right)$, although this may not be nature's choice and much can be found in the literature concerning the Two-HiggsDoublet model (2HDM) [3]. Such extended Higgs sectors arise in many well-motivated extensions of the SM (e.g. Supersymmetric theories).

It may be that higher Higgs representations contribute to symmetry breaking and in this paper we consider an extended Higgs sector proposed by Georgi and Machacek which consists of two Higgs triplets and a Higgs doublet [5] $\rightarrow$ [7]. This model (HTM) preserves $\rho=1$ at tree-level due to a cancellation between the triplet contributions. A fair amount of work has been done the HTM [B] $\rightarrow$ [10], and in this paper we build upon the analysis of our earlier work [11] which considered the phenomenology at LEP2.

Due to the large number of Higgs bosons in the HTM its phenomenology may appear at first sight very complicated. However, we shall see that the degeneracy among the various multiplets, and the constraint on the triplet field vacuum expectation value (VEV) simplify the phenomenology considerably and make the HTM more predictive. In particular, we show that the five-plet members are always heavier than the three-plet members and that the former will always decay dominantly to the latter, giving rise to cascade signatures. For masses in the energy range at LEP2 these decays are often three-body, although due to the fermiophobic nature of the five-plet they still dominate. In addition, we show that the decays of the three-plet bosons to the eigenstate $\psi_{2}$ and a virtual vector boson are possible and may be dominant. The paper is organized as follows. In Section 2 we briefly introduce the HTM and show how precision measurements at LEP constrain its phenomenology. In Section 3 we evaluate limits on the masses of the HTM scalars by using results from present experimental searches for Higgs bosons. In Section 4 we calculate the branching ratios (BRs) of the five-plet and three-plet members for masses relevant for LEP2, and in Section 5 we examine the possible production processes and signatures at this collider. Finally, Section 6 contains our conclusions. 


\section{The Higgs Triplet Model (HTM)}

We do not attempt to give a detailed review of the HTM here and instead refer the reader to Refs. [5] and [8]. The model possesses one isopin doublet (hypercharge $\mathrm{Y}=1$ ) and two triplets with $Y=0$ and $Y=2$ respectively. The particle spectrum consists of a degenerate five-plet of Higgs bosons $\left(H_{5}^{ \pm \pm}, H_{5}^{ \pm}, H_{5}^{0}\right)$, a degenerate three-plet $\left(H_{3}^{ \pm}\right.$, $\left.H_{3}^{0}\right)$, and two singlets under the custodial symmetry $\left(H_{1}^{0}\right.$ and $\left.H_{1}^{0 \prime}\right)$. It is convenient to introduce a doublet-triplet mixing angle $\left(0 \leq \theta_{H} \leq \pi / 2\right)$ defined by

$$
\cos \theta_{H} \equiv \frac{a}{\sqrt{a^{2}+8 b^{2}}}, \quad \sin \theta_{H} \equiv \sqrt{\frac{8 b^{2}}{a^{2}+8 b^{2}}} .
$$

Here $b$ is the VEV of the neutral triplet fields, and $a$ the VEV of the neutral doublet field. The five-plet members and $H_{1}^{0 \prime}$ are composed entirely of triplet fields, and so most tree-level couplings to fermions are forbidden by gauge invariance. The exception is the possibility of $H_{5}^{ \pm \pm}$being coupled to two leptons (see Section 4.2). The threeplet members, $H_{3}^{ \pm}$and $H_{3}^{0}$, are respectively equivalent to $H^{ \pm}$and $A^{0}$ of the $2 \mathrm{HDM}$ (Model I) with the replacement $\cot \beta \rightarrow \tan \theta_{H}$ in the Feynman rules. In the limit of $\tan \theta_{H} \rightarrow 0$ (i.e. the triplet fields do not contribute to symmetry breaking) $H_{1}^{0}$ plays the role of $\phi^{0}$. A full list of Feynman rules for the HTM appears in Ref. [8].

Precision measurements of the process $Z \rightarrow b \bar{b}$ impose the strongest bound on $\sin \theta_{H}$. Virtual charged scalars with tree-level fermion couplings contribute to this decay [12, [13] e.g. $H^{ \pm}$in the $2 \mathrm{HDM}$ and $H_{3}^{ \pm}$in the HTM. Ref. [14 shows that one can obtain the bound $\cot \beta \leq 0.555$ (95\% c.l) in the $2 \mathrm{HDM}$ for $M_{H^{ \pm}}=85 \mathrm{GeV}$, with the bound weakening slightly for larger $M_{H^{ \pm}}$. This corresponds to $\tan \theta_{H}<0.555$ in the HTM, improving the bound $\tan \theta_{H}<0.8$ that we quoted in Ref. [11. Bounds from $B \bar{B}$ mixing can be competitive [15], although suffer from some uncertainties in the measured values of the input parameters. Throughout the paper we shall be using $\tan \theta_{H}<0.555$, which is justified since we are interested in the case of the three-plet being in range at LEP2. We shall see that this constraint on $\tan \theta_{H}$ significantly effects the phenomenology of the HTM, making it more predictive.

We now consider the masses of the Higgs bosons. Mixing may take place between the fields $H_{1}^{0 \prime}$ and $H_{1}^{0}$, and there exist two mass eigenstates denoted by $\psi_{1}$ and $\psi_{2}$ with $M_{\psi_{1}}>M_{\psi_{2}}$. The mass matrix in the basis $H_{1}^{0}-H_{1}^{0 \prime}$ is given by:

$$
M=\left(\begin{array}{cc}
8 c_{H}^{2}\left(\lambda_{1}+\lambda_{3}\right) & 2 \sqrt{6} s_{H} c_{H} \lambda_{3} \\
2 \sqrt{6} s_{H} c_{H} \lambda_{3} & 3 s_{H}^{2}\left(\lambda_{2}+\lambda_{3}\right)
\end{array}\right) v^{2} .
$$

The compositions of the mass eigenstates are:

$$
\begin{aligned}
& \psi_{1}=H_{1}^{0^{\prime}} \sin \alpha+H_{1}^{0} \cos \alpha, \\
& \psi_{2}=H_{1}^{0^{\prime}} \cos \alpha-H_{1}^{0} \sin \alpha .
\end{aligned}
$$


We shall denote the common mass of the three-plet (five-plet) members as $M_{3}\left(M_{5}\right)$, with values given by:

$$
M_{3}^{2}=\lambda_{4} v^{2} \quad M_{5}^{2}=3\left(s_{H}^{2} \lambda_{5}+c_{H}^{2} \lambda_{4}\right) .
$$

Here $\lambda_{i}$ are parameters from the Higgs potential, $s_{H}=\sin \theta_{H}$ and $c_{H}=\cos \theta_{H}$. If one allows $s_{H}^{2} \rightarrow 1$ (i.e. no constraint on $s_{H}$ ), which is often taken in the literature to maximize the effects of exotic couplings that depend on $s_{H}^{2}$, there would exist a parameter space for $M_{5} \leq M_{3}$. Assuming that the five-plet members are the lightest, Refs. [8], [16] $\rightarrow$ 18] consider the decay channels of $H_{5}^{0}, H_{5}^{ \pm \pm}$and $H_{5}^{ \pm}$. They conclude that for $M_{5}$ in range at LEP2 one would find $H_{5}^{ \pm \pm} \rightarrow W^{(*)} W^{*}, H_{5}^{ \pm} \rightarrow W^{(*)} Z^{*}$, and $H_{5}^{0} \rightarrow \gamma \gamma$ as the dominant decays. However, in the light of the bound on $s_{H}^{2}$ one finds that the five-plet members are always heavier than the three-plet members. Even equating $\lambda_{5}=0$ and $c_{H}^{2}$ at 0.764 (its lowest value) one would find from Eq. (5) that $M_{5} \approx 1.5 M_{3}$. This result has important consequences for the phenomenology of the five-plet members, since decays to $H_{3} H_{3}$ or $H_{3} V$ ( $V$ is $W$ or $Z$ ) will always be available, with sometimes one of the particles off-shell. We will show that in the HTM these three-body decays to lighter Higgs bosons dominant the decays to two vector bosons for $M_{5}$ in range at LEP2, and so would imply cascade decay signatures for the five-plet.2 A recent search at LEP2 for $H_{5}^{0}$ [19 assumed the decays $H_{5}^{0} \rightarrow H_{3} V^{*}$ to be negligible and obtained the bound $M_{5} \geq 79.5 \mathrm{GeV}$.

The masses of $\psi_{1}$ and $\psi_{2}$ are dependent on $\lambda_{1}, \lambda_{2}, \lambda_{3}$, and so there is no correlation between $M_{\psi_{1}}, M_{\psi_{2}}$ and $M_{3}, M_{5}$. Therefore one can consider the case of $\psi_{2}$ being lighter than $M_{3}$. In Ref. [11] we showed that a natural argument of taking all $\lambda_{i}$ equal suggested $\psi_{2}$ to be the lightest of the Higgs bosons. It was also shown that very little mixing occurs and that one may take $H_{1}^{0}$ and $H_{1}^{0 \prime}$ to be effectively mass eigenstates. With the improved bound $s_{H}^{2} \leq 0.236$ one would find the mixing angle $\alpha$ constrained even more.

We shall assume that $\psi_{1}$ is the heaviest of the Higgs bosons and is out of the LEP2 range - the natural argument in Ref. [11] would suggest this. We shall consider two scenarios, bearing in mind that $M_{3} \leq M_{5}$.

(i) $M_{\psi_{2}} \leq M_{3}$

(ii) The three-plet members are the lightest of the Higgs bosons

These two situations may produce different experimental signatures, since in case (i) we expect a parameter space for significant three body decays of the three-plet to $\psi_{2}$ (in an analogous way to the results in the 2HDM [20]). In case (ii) we expect the three-plet BRs to be identical to those of the 2HDM (Model I).

\footnotetext{
${ }^{2}$ The results of Refs. [8], [16] $\rightarrow[18]$ will still apply to other models in which a fermiophobic $H^{ \pm \pm}$, $H^{ \pm}$or $H^{0}$ exists.
} 


\section{$3 \quad$ Experimental limits on masses}

In this section we derive mass limits on the Higgs bosons of the HTM by using current experimental limits valid for $\phi^{0}$ and the scalars of the 2HDM. First we consider $\psi_{2}$. If one assumes $\psi_{2}=H_{1}^{0}$ (i.e. if $\cos \alpha=0$ in Eq. (4)) one may use the current LEP searches for $\phi^{0}$ to place a direct mass bound since $H_{1}^{0}$ has essentially the same BRs as $\phi^{0}$ for masses in range at LEP; although its fermion couplings are scaled by a factor $1 / c_{H}$ and the vector boson couplings by a factor $c_{H}$ relative to $\phi^{0}$, since decays to the latter are negligible at LEP energies, one can apply the results from $\phi^{0}$ searches. Hence one obtains $H_{1}^{0} \geq 87.6 \mathrm{GeV}$ for $c_{H}^{2}=1$ [21] (i.e. the $H_{1}^{0} Z Z$ coupling is $\phi^{0} Z Z$ strength). For the smallest value of $c_{H}\left(c_{H}^{2} \geq 0.764\right)$ one would find a limit of $\approx 69.5 \mathrm{GeV}$ [22].

If $\psi_{2}=H_{1}^{0 \prime}$, (i.e. $\left.\sin \alpha=0\right) \psi_{2}$ would be a fermiophobic Higgs $\left(H_{F}\right)$ [23] and one would expect a very large $\mathrm{BR}$ to $\gamma \gamma$. Using the production mechanism $e^{+} e^{-} \rightarrow H_{F} Z$, and assuming that the cross-section is equal to that of $e^{+} e^{-} \rightarrow \phi^{0} Z$, Ref. [19] obtain the limit $M_{F} \geq 90 \mathrm{GeV}$. For $H_{1}^{0 \prime}$ this limit may be weakened since the coupling $H_{1}^{0 \prime} Z Z$ (which determines the cross-section) is proportional to $8 s_{H}^{2} / 3$. For small $s_{H}$ a very light $H_{1}^{0 \prime}$ could still be possible, and would be a 'hidden' fermiophobic Higgs. We note here that Ref. [19] also searches for $\gamma \gamma f \bar{f}$ final states, without requiring that the fermions originate from a $Z$. Therefore this search is sensitive to the process $e^{+} e^{-} \rightarrow \psi_{2} H_{3}^{0}$, with $H_{3}^{0} \rightarrow f \bar{f}$. Ref. [19] shows that the sum of the cross-sections must satisfy the following relation:

$$
\sigma\left(e^{+} e^{-} \rightarrow \psi_{2} Z\right)+\sigma\left(e^{+} e^{-} \rightarrow \psi_{2} H_{3}^{0}\right) \leq 150 \mathrm{fb}
$$

Since these two production processes are complementary, if one wishes to consider a light fermiophobic $\psi_{2}$ Eq. (6) can only be satisfied if $e^{+} e^{-} \rightarrow \psi_{2} H_{3}^{0}$ is closed/suppressed - i.e. $M_{\psi_{2}}+M_{3} \geq 160 \mathrm{GeV}$. A caveat here is that in these scenarios of a light $\psi_{2}$ one would have a large parameter space for a significant/dominant $\operatorname{BR}\left(H_{3}^{0} \rightarrow Z^{(*)} \psi_{2}\right)$, see Section 4.1. Although this would produce a different event topology $(\gamma \gamma$ recoiling against $\gamma \gamma f \bar{f})$ much of the current selection criteria would still be relevant [24]. We conclude that a light fermiophobic $\psi_{2}$ would most likely require $M_{\psi_{2}}+M_{3} \geq 160 \mathrm{GeV}$, although $M_{\psi_{2}}+M_{3} \leq 160 \mathrm{GeV}$ is not strictly ruled out.

In the general case of $\psi_{2}$ being a mixture of the above two states (i.e. if $\lambda_{3} \neq 0$ and $s_{H} \neq 0$ ), one finds the coupling $Z Z \psi_{2}$ relative to that for $Z Z \phi^{0}$ to be:

$$
Z Z \psi_{2}=\frac{2 \sqrt{2}}{\sqrt{3}} \cos \alpha s_{H}-\sin \alpha c_{H} .
$$

The mixing angle $\alpha$ is found from:

$$
\sin 2 \alpha=\frac{2 M_{12}}{\sqrt{\left(M_{11}-M_{22}\right)^{2}+4 M_{12}^{2}}} .
$$


Here $M_{i j}$ are the mass matrix entries in Eq. (2). The angle $\alpha$ may take values from $-\pi / 2 \rightarrow \pi / 2$ i.e. negative values if $M_{12}$ is negative $\left(\lambda_{3}<0\right) . \quad \lambda_{3}$ may be positive or negative, in contrast to $\lambda_{4}$ and $\lambda_{5}$ which must be positive ([7). Ref. [10] plotted the coupling $Z Z \psi_{2}$ as a function of $t_{H}^{2}$, allowing values of the latter up to 6.25 . We are interested in the region $t_{H}^{2} \leq 0.31$ which is consequently not very clear in the graphs in Ref. [10]. In the light of the bound on $t_{H}$, we find it more appealing to plot the coupling in Eq. (7) as a function of $\alpha$, which was chosen to have fixed values in Ref. [10]. We draw two curves for $\left|Z Z \psi_{2}\right|^{2}$ corresponding to $t_{H}=0.555$ (maximum value) and 0.3 , and one can clearly see not only the destructive interference for positive $\alpha$, but also the constructive interference which was not noticed in Ref. [10. Thus in the general case of mixing, $\psi_{2}$ may attain larger cross-sections than are possible for either of the individual fields $H_{1}^{0 \prime}$ and $H_{1}^{0}$. Such an enhancement is never possible in extended models with only doublets, due to the familiar suppression factors $\sin ^{2}(\beta-\alpha)$ and $\cos ^{2}(\beta-\alpha)$. In the case of destructive interference a very light $\psi_{2}$ is not ruled out. Hence it is possible that $\psi_{2}$ could be light, and the presence of a light $\psi_{2}$ can drastically affect the decay channels of the three-plet bosons (see Section 4.1).

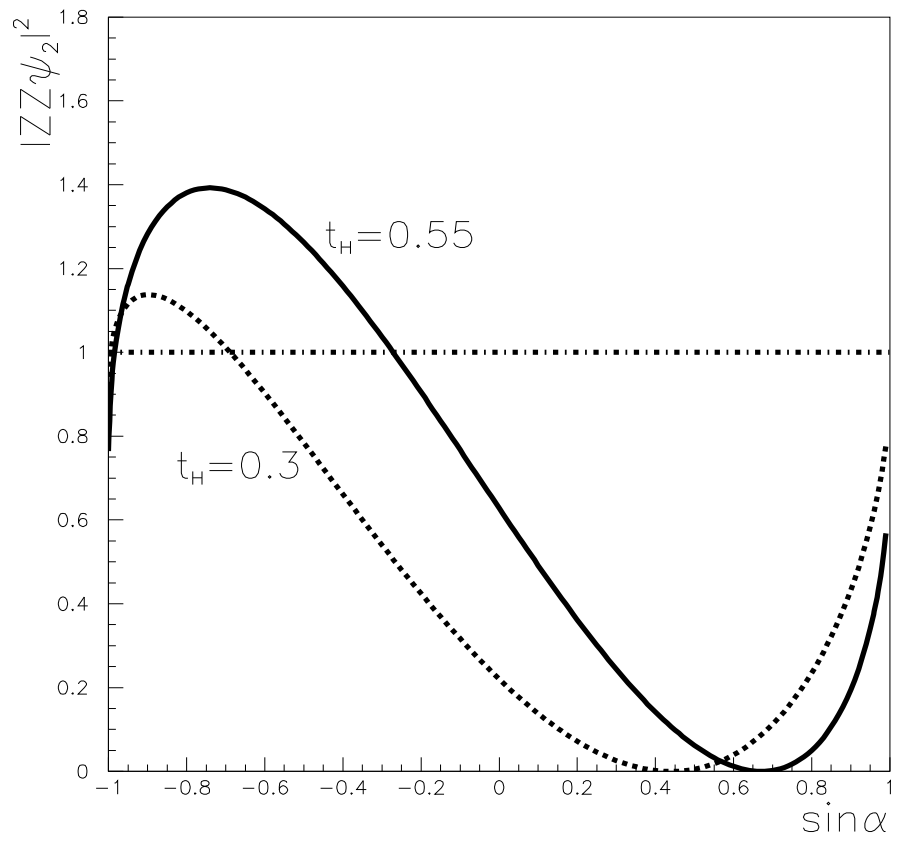

Figure 1: Coupling $\left|Z Z \psi_{2}\right|^{2}$ relative to that of $\left|Z Z \phi^{0}\right|^{2}$ against $\sin \alpha$.

In Fig. 2 we plot $\operatorname{BR}\left(\psi_{2} \rightarrow \gamma \gamma\right)$ as a function of $\sin \alpha$ for three values of $t_{H}$. The decays of $\psi_{2}$ are dominated by the component of $H_{1}^{0}$ unless $\sin \alpha$ is small which corre- 
sponds to a large component of $H_{1}^{0 \prime}$. We recall that the natural scenario considered in Ref. [11] shows that such tiny mixing would be expected, and that $\sin \alpha \leq 0.05$ would occur in the case of all $\lambda_{i}$ equal.

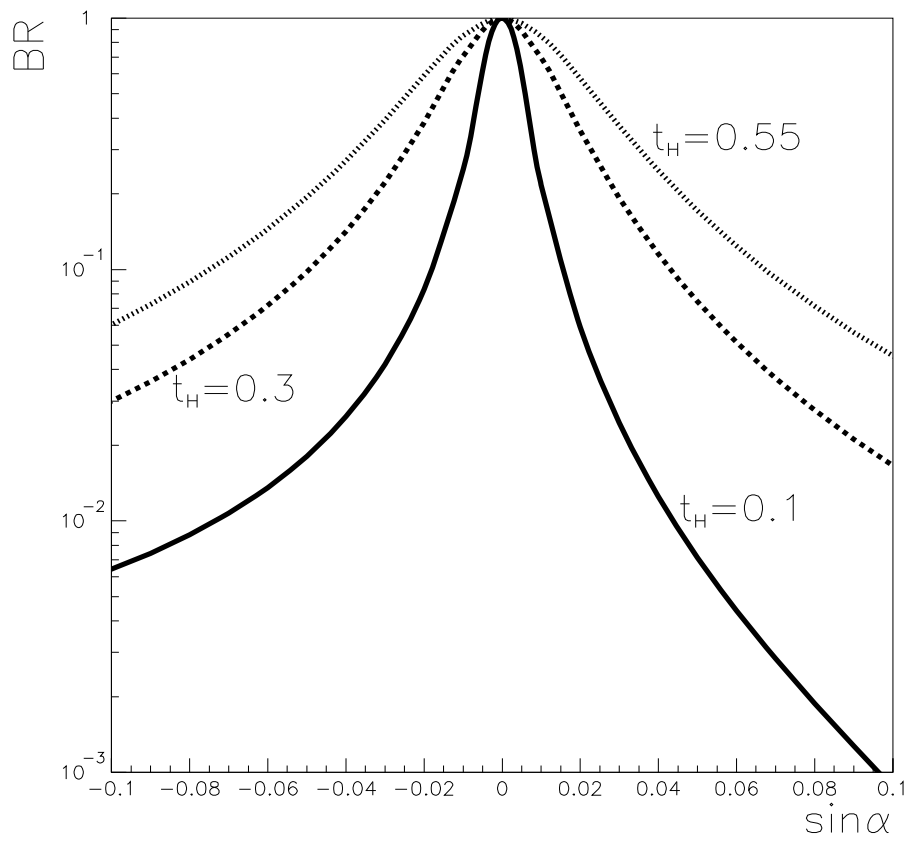

Figure 2: $\mathrm{BR}\left(\psi_{2} \rightarrow \gamma \gamma\right)$ against $\sin \alpha$, for $M_{\psi_{2}}=70 \mathrm{GeV}$.

For the three-plet bosons there are no direct mass limits on $H_{3}^{0}$ independent of $M_{\psi_{2}}$, in the same way that no bound on $A^{0}$ exists independent of $M_{h^{0}}$ in the general 2HDM (apart from the bound $M_{A} \geq 5 \mathrm{GeV}$ from considering the decay $\Upsilon \rightarrow A^{0} \gamma$ [25]). In the HTM one could use the bound $M_{3}+M_{\psi_{2}} \geq 90-110 \mathrm{GeV}$ which is obtained from the combined search for $h^{0}$ and $A^{0}$ [26]. A caveat here is that the cross-sections for $e^{+} e^{-} \rightarrow Z \psi_{2}$ and $e^{+} e^{-} \rightarrow H_{3}^{0} \psi_{2}$ may be larger than in 2HDM (see Section 5) and so the bound on $M_{3}+M_{\psi_{2}}$ could be increased. Since $H_{3}^{0}$ is degenerate with $H_{3}^{ \pm}$, any bound on the latter could be used for the former. Current charged Higgs boson searches assume decays to $\tau \nu_{\tau}$ or $c s$ and obtain the lower bound of $55 \mathrm{GeV}$ (95\% c.l) [27]. $H_{3}^{ \pm}$would decay in this way in the absence of a light $\psi_{2}$. In the 2HDM (Model I) the existence of a light neutral Higgs can invalidate this limit, although this is not the case in HTM since the bound $M_{3}+M_{\psi_{2}} \geq 90-110 \mathrm{GeV}$ also applies to $H_{3}^{ \pm}$, thus ruling out the possibility of $M_{\psi_{2}} \leq M_{3} \leq 55 \mathrm{GeV}$.

For the five-plet there again exist no direct limits since, as we shall show, these bosons would decay via cascades to the three-plet members, giving final states that 
have not been searched for. However, since the five-plet is heavier than the three-plet, one may obtain the bound $M_{5} \geq 82.5 \mathrm{GeV}$, found by multiplying the bound on $M_{3}$ by 1.5 .

\section{Branching ratios}

In this section we study the branching ratios of the five-plet members and the threeplet members. The possible decays for the five-plet will involve $H_{5} \rightarrow H_{3} H_{3}$ and $H_{3} V$, where one of the particles may be off-shell for masses in range at LEP2 energies. If $\psi_{2}$ were lighter than the three-plet then the decay $H_{3} \rightarrow \psi_{2} V^{*}$ would be possible. Ref. [8] considers the decays of the five-plet to the three-plet, although for mass choices that are not relevant for LEP2 i.e. they take $M_{3}=81 \mathrm{GeV}$, which would give $M_{5}=121.5$ $\mathrm{GeV}$, thus taking the five-plet out of range. In addition, $t_{H}^{2}$ was fixed to the values of $0.01,2.25$ and 100, while we wish to concentrate on the region $t_{H}^{2} \leq 0.31$. We aim to give branching ratios for these channels for masses in range at LEP2, making use of the fact that $M_{5} \geq 1.5 M_{3}$ and so the vector boson is never very off-shell in the decay $H_{5} \rightarrow H_{3} V^{*}$. In addition, Ref. [8] did not consider the possibility of the decay $H_{3} \rightarrow \psi_{2} V^{*}$.

In the following subsections we shall be neglecting the decays $H_{5} \rightarrow H_{3}^{*} V$ i.e. where the Higgs boson is off-shell and the vector boson is on-shell. Ref. [8] explains that these are very small compared to $H_{5} \rightarrow H_{3} V^{*}$ since $V^{*} \rightarrow f \bar{f}$ is a gauge strength coupling while $H_{3}^{*} \rightarrow f \bar{f}$ is Yukawa strength; moreover, the latter involves $t_{H}$ which can never compensate for the smallness of the coupling.

\subsection{The decays of the three-plet to $\psi_{2}$}

In this subsection we consider the decays $H_{3}^{0} \rightarrow Z^{*} \psi_{2}$ and $H_{3}^{ \pm} \rightarrow W^{*} \psi_{2}$. Analogous decays of $H^{ \pm}$and $A^{0}$ in the 2HDM (Model I) were shown to be dominant over a wide range of parameter space in Ref. [20]. In the HTM, $\psi_{2}$ is a mixture of $H_{1}^{0}$ and $H_{1}^{0 \prime}$ and the width for this three-body decay is given by [28], 29]:

$$
\begin{gathered}
\Gamma\left(H_{3}^{ \pm} \rightarrow \psi_{2} W^{*} \rightarrow h f \bar{f}^{\prime}\right)=\frac{9 G_{F}^{2} M_{W}^{4}}{16 \pi^{3}} M_{H^{ \pm}} C G_{\psi W}, \\
\Gamma\left(H_{3}^{0} \rightarrow \psi_{2} Z^{*} \rightarrow h f \bar{f}\right)=\frac{6.1 G_{F}^{2} M_{W}^{4}}{8 \pi^{3}} M_{H^{ \pm}} C G_{\psi Z} .
\end{gathered}
$$

The analytical form of the function $G_{\psi V}$ may be used for $M_{3} \geq M_{W}$. For $M_{3} \leq M_{W}$ one must perform the integral numerically. The coupling $C$ is given by

$$
C=\frac{8}{3} \cos ^{2} \alpha c_{H}^{2}+\sin ^{2} \alpha s_{H}^{2}+\frac{4 \sqrt{2}}{\sqrt{3}} s_{H} c_{H} \sin \alpha \cos \alpha .
$$




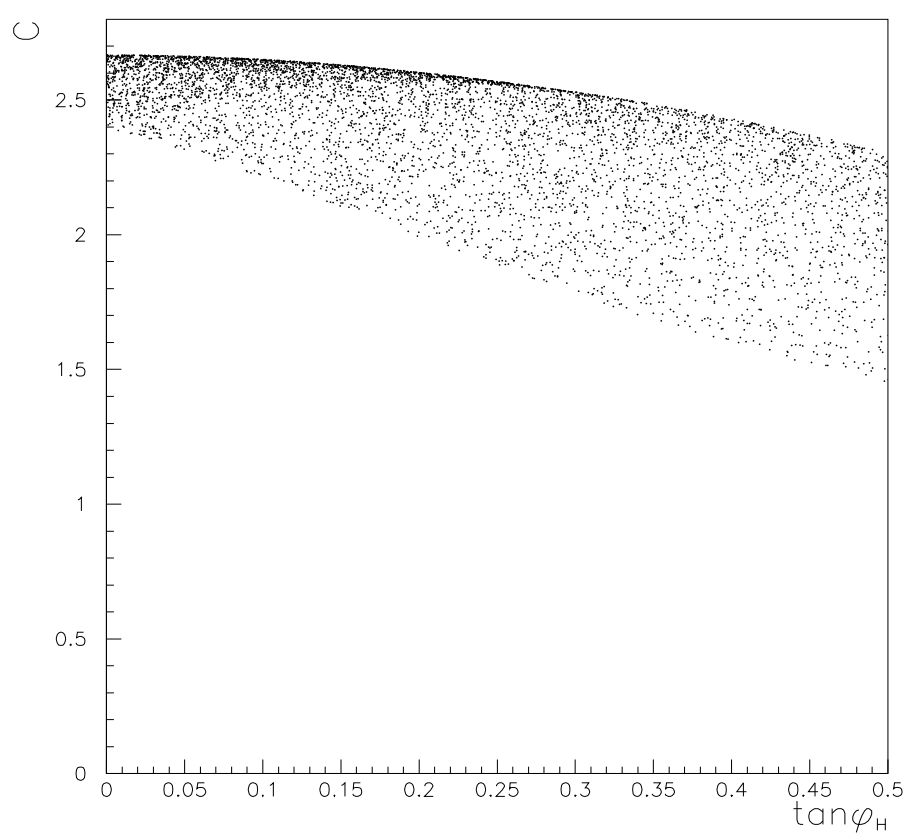

Figure 3: The coupling $C$ as a function of $t_{H}$, demanding a light $\psi_{2}$.

In order to allow a very light $\psi_{2}$ which has escaped detection at LEP one must impose a condition on the $Z Z \psi_{2}$ coupling (Eq. (7)). For example, for $M_{\psi_{2}} \leq 34 \mathrm{GeV}$ one requires $\sigma\left(e^{+} e^{-} \rightarrow Z^{*} \psi_{2}\right) \leq 0.1 \sigma\left(e^{+} e^{-} \rightarrow Z^{*} \phi^{0}\right)$ [22]. Imposing this condition we plot in Fig. 3 the value of $C$ as a function of $t_{H}$ for 5000 random values of $\alpha$ and $\theta$. We see that smaller $t_{H}$ causes larger $C$, and so decreasing $t_{H}$ enhances the three-body width and simultaneously reduces the widths of the competing decays, since $\Gamma\left(H_{3} \rightarrow f \bar{f}\right)$ is proportional to $t_{H}^{2}$. We also note that the value of $C$ is always greater than 1 , and so the relative strength of these three-body decays is greater than those for the analogous decays in the 2HDM (Model I) in which $C=1$ or $\cos ^{2}(\beta-\alpha)$. Hence in order to see their magnitude in the HTM it is sufficient to use the figures in Ref. 20] bearing in mind that the widths in the HTM will be greater by a factor $C$ i.e. use Figs. 2,3 for $H_{3}^{ \pm}$and Fig. 7 for $H_{3}^{0}$ with appropriate scaling and interpreting $\tan \beta$ as $\cot \theta_{H}$. We note that these decays of the three-plet bosons were not considered in Ref. [8], although may be dominant or even close to $100 \%$ over a wide range of parameter space. In addition, they would be an alternative way of producing a very light $\psi_{2}$ which is escaping current searches. 


\subsection{The decays of $H_{5}^{ \pm \pm}$}

The decays we shall consider are the following:

(i) $H_{5}^{ \pm \pm} \rightarrow W W^{*}$

(ii) $H_{5}^{ \pm \pm} \rightarrow H_{3}^{ \pm} W^{*}$

(iii) $H_{5}^{ \pm \pm} \rightarrow H_{3}^{ \pm} H_{3}^{ \pm}$

There is another possible decay, that is, $H_{5}^{ \pm \pm} \rightarrow l^{ \pm} l^{ \pm}$(for recent work see Refs. 30, [31). In the HTM these decays are only significant if $s_{H}$ is very small, since the width is given by

$$
\Gamma\left(H_{5}^{ \pm \pm} \rightarrow l^{ \pm} l^{ \pm}\right)=\frac{\left|h_{l l}\right|^{2}}{8 \pi} M_{5}
$$

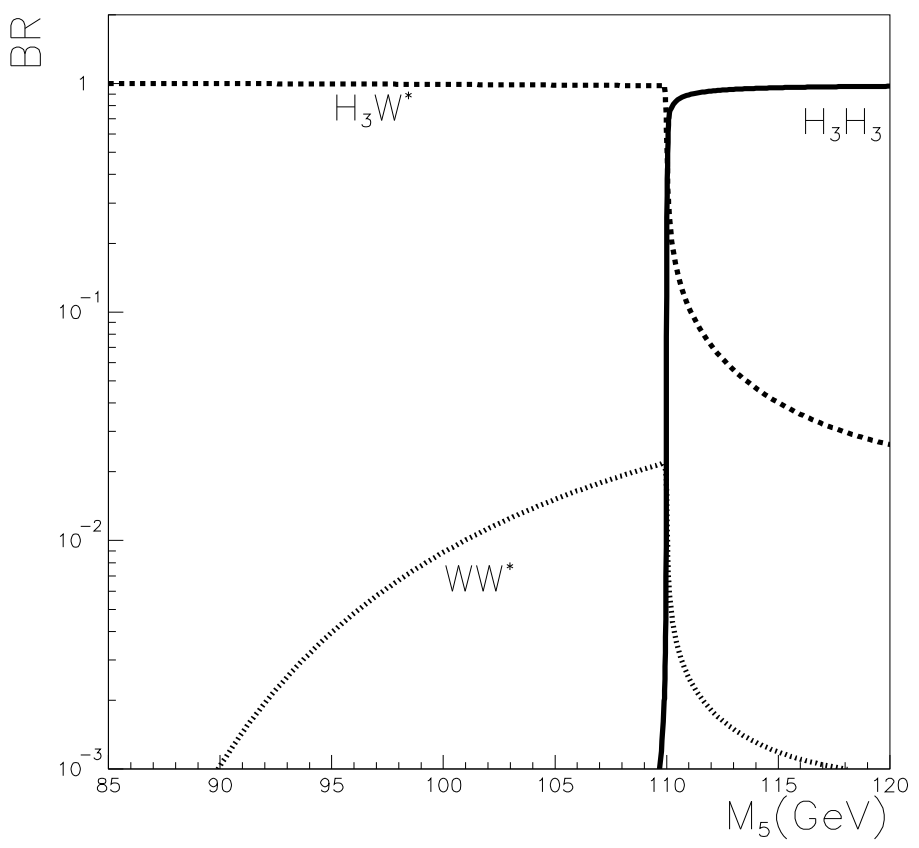

Figure 4: BRs of $H_{5}^{ \pm \pm}$with $\tan \theta_{H}=0.555$.

The current neutrino mass limits constrain $h_{\tau \tau} \leq 1.4 \times 10^{-4} / s_{H}$, while the couplings $h_{\mu \mu}$ and $h_{e e}$ are smaller by 3 orders and 8 orders of magnitude respectively. Ref. 30 considered the decays of an $H^{ \pm \pm}$in a model with a Higgs doublet and only one triplet. They considered decays (i) and (ii) and the bi-lepton channels are always strong since 
$s_{H} \leq 0.0056$ (95\% c.l) in this model. We shall neglect the bi-lepton channels since in the HTM since one usually considers larger values of $s_{H}$. Ref. [30] found that the decay (ii) is important, and we find the same. Importantly, the HTM requires $M_{5} \geq M_{3}$ and so this channel is always open, and we also expect the bi-lepton channel to be small. In Fig. 3 we plot the BRs for the channels (i), (ii) and (iii) as a function of $M_{5}$, fixing $M_{3}=55 \mathrm{GeV}$ (i.e. its lower limit) and $t_{H}=0.555$. We see that the three-body decay (ii) is close to $100 \%$ until the real threshold for $H_{3} H_{3}$ decays is reached. Beyond this threshold the decay (iii) dominates. We note that the decay to two vector bosons does not surpass a BR of $2 \%$. The domination of channel (ii) before the real $\mathrm{H}_{3} \mathrm{H}_{3}$ threshold is reached is due to two reasons; the decay is not severely phase space suppressed since $M_{5} \geq 1.5 M_{3}$, and the coupling $H_{5}^{ \pm \pm} H_{3}^{ \pm} W$ is proportional to $c_{H}$. The off-shell decay $H_{3} H_{3}^{*}$ is included in our plots although is small. Lowering the value of $t_{H}$ causes the decay (iii) to reach $100 \%$ more quickly, and further reduces decay (i).

\subsection{The decays of $H_{5}^{ \pm}$}

The decays we shall consider are the following:

(i) $H_{5}^{ \pm} \rightarrow Z^{*} W^{(*)}$

(ii) $H_{5}^{ \pm} \rightarrow H_{3}^{ \pm} Z^{*}$

(iii) $H_{5}^{ \pm} \rightarrow H_{3}^{0} W^{*}$

(iv) $H_{5}^{ \pm} \rightarrow H_{3}^{0} H_{3}^{ \pm}$

Here there are two possible decays to a three-plet member and a vector boson, (ii) and (iii), and decay (i) is not possible in models with only Higgs doublets. The width of decay (i) was calculated in Ref. [17] although here we will see that this channel has a much smaller width than the decays (ii) and (iii). In Fig. 4 we plot the analogy of Fig. 3 for $H_{5}^{ \pm}$. Decay (i), which would be the dominate channel if $H_{5}^{ \pm}$were the lightest, is not included in our plots although would peak at $\mathrm{BR} \approx 0.3 \%$ in the region just before $M_{5}=110 \mathrm{GeV}$. Again the three-body decays to the three-plet and a virtual vector boson dominate until the real $\mathrm{H}_{3} \mathrm{H}_{3}$ threshold is reached. We see that the decay mediated via $W^{*}$ is stronger than that mediated via $Z^{*}$. This is due to the fact that for a given $M_{5}$ and $M_{3}, Z$ is more off-shell than $W$, which compensates for the slightly weaker couplings of the $W$ mediated decay in Eq. (9).

\subsection{The decays of $H_{5}^{0}$}

The decays we shall consider are the following:

(i) $H_{5}^{0} \rightarrow H_{3}^{ \pm} H_{3}^{\mp}$ 


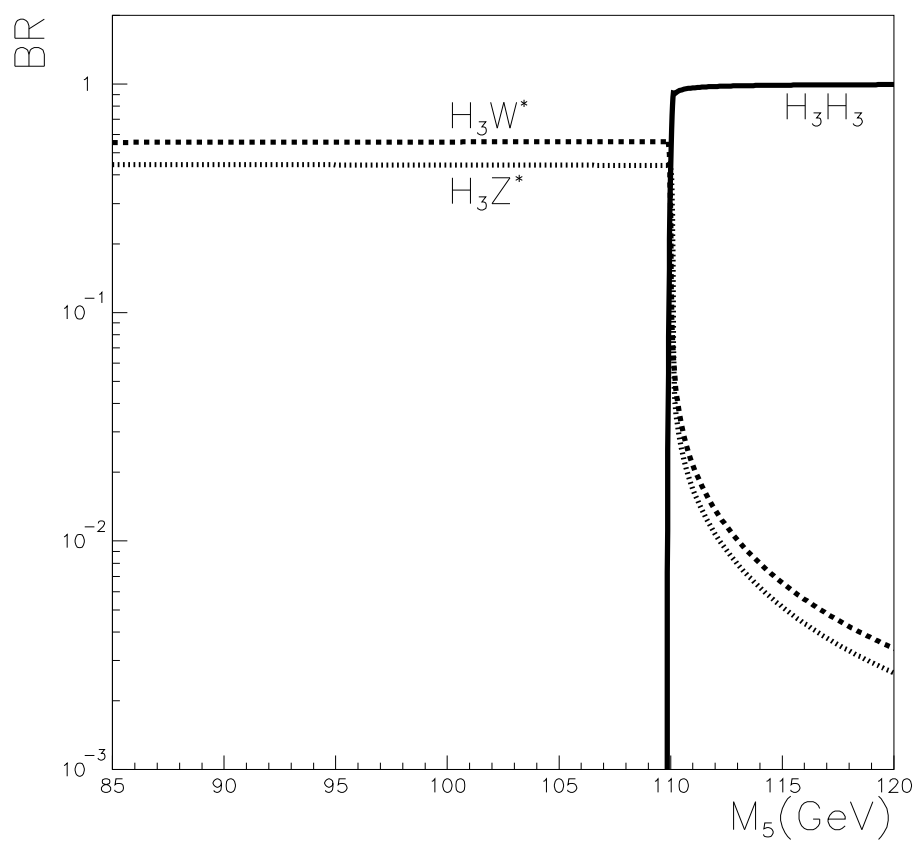

Figure 5: Same as Fig. 4 but for $H_{5}^{ \pm}$.

(ii) $H_{5}^{0} \rightarrow H_{3}^{0} H_{3}^{0}$

(iii) $H_{5}^{0} \rightarrow H_{3}^{ \pm} W^{*}$

(iv) $H_{5}^{0} \rightarrow H_{3}^{0} Z^{*}$

(v) $H_{5}^{0} \rightarrow \gamma \gamma, W^{*} W^{(*)}, Z^{*} Z^{(*)}$

In the absence of decays to the three-plet $H_{5}^{0}$ would decay predominantly (for $M_{5} \leq 90$ $\mathrm{GeV}$ ) to a mixture of $\gamma \gamma$ and $f \bar{f} 1$-loop induced decays 8 . In Fig. 5 we plot the analogy of Fig. 3 for $H_{5}^{0}$. Again, decays (iii) and (iv) share the domination until the real threshold for decays (i) and (ii) is reached. We note that the $Z^{*}$ mediated decay is stronger than that mediated by $W^{*}$, in contrast to case for $H_{5}^{ \pm}$; this is due to the fact that the coupling $H_{5}^{0} H_{3}^{0} Z$ contains an extra factor of two with respect to the coupling $H_{5}^{0} \rightarrow H_{3}^{ \pm} W$. In the region $M_{5} \geq 2 M_{3}$, decay (ii) has partial width four times greater than that of decay (i). The BR to $\gamma \gamma$ is reduced to values less than $2 \%$, and this result would effect current search techniques [19] which only assume decay channels (v). 


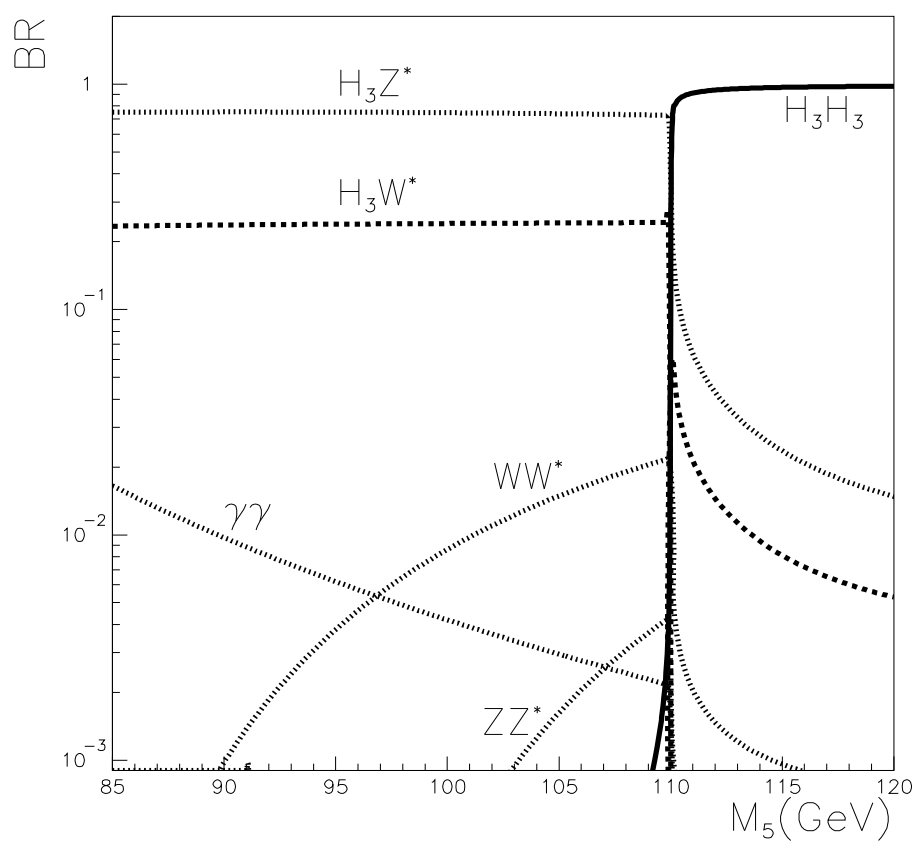

Figure 6: Same as Fig. 4 but for $H_{5}^{0}$.

\section{Production Channels at LEP2}

In this section we consider the possible signatures of the Higgs bosons of the HTM at LEP2. Some of the production channels are analogies of production channels in models with only Higgs doublets, while others are particular to models with higher representations. In the charged Higgs sector one has:

$$
e^{+} e^{-} \rightarrow H_{3}^{+} H_{3}^{-}, H_{5}^{+} H_{5}^{-}, H_{5}^{++} H_{5}^{--}, H_{3}^{+} H_{5}^{-}, W^{ \pm} H_{5}^{\mp} \text {. }
$$

Identical pair production (the first three) has received a lot of coverage in the literature [8], 32, while the last two are not possible in models with only doublets. The mechanism $e^{+} e^{-} \rightarrow W^{\mp} H_{5}^{ \pm}$has received some attention in the literature [17], [33] although suffers from the suppression factor $s_{H}^{2}$. Non-identical pair production can be sizeable if kinematically allowed, being proportional to $c_{H}^{2}$ and having a permutation factor of 2. In addition, it can be open when the pair production of the five-plet bosons is not. In the neutral sector one has the bremsstrahlung channels:

$$
e^{+} e^{-} \rightarrow Z H_{5}^{0}, Z \psi_{2}
$$


and the pair production channels:

$$
e^{+} e^{-} \rightarrow H_{3}^{0} H_{5}^{0}, H_{3}^{0} \psi_{2}
$$

The phenomenology of the three-plet would look very similar to that of the 2HDM (Model I) since $H_{3}^{0}$ and $H_{3}^{ \pm}$have the same couplings as $A^{0}$ and $H^{ \pm}$respectively, with the replacement $\cot \beta \rightarrow \tan \theta_{H}$. If there exists a light $\psi_{2}$ then one may use the analysis in Ref. [20], bearing in mind the decays of the three-plet to $\psi_{2}$ (Section 4.1) may be stronger than the analogous decay in the 2HDM. In addition, the mechanism $e^{+} e^{-} \rightarrow \psi_{2} H_{3}^{0}$, being proportional to $C$ in Eq. (9), can have a larger cross-section than that for $e^{+} e^{-} \rightarrow A^{0} h$ in the 2HDM; this is also the case for $e^{+} e^{-} \rightarrow Z \psi_{2}$ (see Fig. 1).

If the five-plet is in range one would expect high-multiplicity cascade signatures. In Table 1 we outline the possible signatures of the five-plet members from the various production mechanisms. We assume $\sqrt{s}=192 \mathrm{GeV}$ and the entries in brackets allow the additional cascade decay $H_{3} \rightarrow \psi_{2} V^{*}$; 'not open' indicates that the production channel in question would not be kinematically allowed.

\begin{tabular}{|c|c|c|}
\hline & $H_{5} \rightarrow H_{3} V^{*}$ & $H_{5} \rightarrow H_{3} H_{3}$ \\
\hline$H_{5}^{++} H_{5}^{--}$ & 8f (12f) & not open \\
\hline$H_{5}^{+} H_{5}^{-}$ & 8f (12f) & not open \\
\hline$H_{5}^{0} Z$ & 6f (8f) & not open \\
\hline$H_{5}^{ \pm} W$ & 6f (8f) & 6f (10f) \\
\hline$H_{5}^{ \pm} H_{3}^{\mp}$ & 6f (10f) & 6f (12f) \\
\hline$H_{5}^{0} H_{3}^{0}$ & 6f (10f) & 6f (12f) \\
\hline
\end{tabular}

Table 1: The signatures of the five-plet members.

The combination of the constraints $M_{5} \geq 1.5 M_{3}$ and $M_{\psi_{2}}+M_{3} \geq 110 \mathrm{GeV}$ means that there is only a small parameter space open at LEP2 which allows the cascade decay of $H_{5} \rightarrow \psi_{2} X$ via the three-plet to be open. For example, mass choices such as $M_{5}=90 \mathrm{GeV}, M_{3}=60 \mathrm{GeV}$ and $M_{\psi_{2}}=50 \mathrm{GeV}$ would allow pair production of the five-plet to be open and satisfy the mass relations above; in these cases the difference between $M_{3}$ and $M_{\psi_{2}}$ is small and so one would need smaller $\tan \theta_{H}$ in order to allow a substantial BR for the three-body decay $H_{3} \rightarrow \psi_{2} V^{*}$. We note that for single production of $H_{5}^{ \pm}, H_{5}^{0}$ in association with a gauge boson one could find asymmetric topologies such as 2 fermions recoiling against 6 or 8 fermions. For the signatures in the first column one can always trigger on leptons originating from the virtual vector bosons. Jets originating from $H_{3}$ could be reconstructed to give the mass of the three-plet bosons. We conclude that the signature of the five-plet would be a large multiplicity fermion event. 


\section{Conclusions}

We have studied the phenomenology of the 2 triplet, 1 doublet model (HTM) in the context of LEP2. We showed that current precision measurements tightly constrain the triplet-doublet VEV ratio, and so cause the five-plet $\left(H_{5}\right)$ bosons to be heavier than the three-plet $\left(H_{3}\right)$. We found that the $\mathrm{BR}\left(H_{5} \rightarrow H_{3} V^{*}\right) \approx 100 \%$ until the real threshold for $H_{5} \rightarrow H_{3} H_{3}$ decays is reached. This ensures that the signature of the five-plet bosons (if in range at LEP2) would be a large multiplicity fermion event. We also showed that $\psi_{2}$ may possess a production cross-section at LEP2 up to 1.4 times that of the minimal standard model Higgs. Such an enhancement is never possible in models with only doublet representations. Conversely, $\psi_{2}$ may also be very weakly coupled to the $Z$ which would allow a light $\psi_{2}$ to have eluded detection at LEP. In this case the decays $H_{3} \rightarrow \psi_{2} V^{*}$ are allowed and we showed that they may be the dominant channel over a wide region of parameter space, a result that would affect current search techniques for the three-plet and be an alternative way of searching for a weakly coupled $\psi_{2}$.

\section{Acknowledgements}

This work was supported by DGICYT under grants PB95-1077, by the TMR network grant ERBFMRXCT960090 of the European Union, and by a CSIC-UK Royal Society fellowship. I wish to thank A. Turcot for useful comments and for proofreading the article.

\section{References}

[1] S. Weinberg, Phys. Rev. Lett. 19 (1967) 1264; S. Glashow, Nucl. Phys. 20, (1961) 579; A. Salam, in Elementary Particle Theory, ed. N. Svartholm, (1968) p367.

[2] P.W. Higgs, Phys. Lett. B12 (1964) 132, Phys. Rev. Lett. 13 (1964) 508.

[3] J.F. Gunion, H.E. Haber, G.L. Kane and S. Dawson, The Higgs Hunter's Guide (Addison-Wesley, Reading, 1990).

[4] Review of Particle Properties, Phys. Rev. D50 (1996).

[5] H. Georgi and M. Machacek, Nucl. Phys. B262 (1985) 463.

[6] R.S. Chivukula and H. Georgi, Phys. Lett. B182 (1986) 181.

[7] M.S. Chanowitz and M. Golden, Phys. Lett. B165 (1985) 105. 
[8] J.F. Gunion, R. Vega, and J. Wudka, Phys. Rev. D42 (1990) 1673; Phys. Rev. D43 (1991) 2322.

[9] R. Vega and D.A. Dicus, Nucl. Phys. B329 (1990) 533.

[10] P. Bamert and Z. Kunst, Phys. Lett. B306 (1993) 335.

[11] A.G. Akeroyd, Phys. Lett. B353 (1995) 519.

[12] A. Denner, R.J. Guth, W. Hollik and J.H. Kuhn, Z. Phys. B51 (1991) 695.

[13] A. Kundu and B. Mukhopadhyaya, Int. J. Mod. Phys. A11 (1996) 5221.

[14] M. Ciuchini, G. Degrassi, P. Gambino and G.F. Giudice, Nucl. Phys. B527 (1998) 527.

[15] D. Chakraverty and A. Kundu, Mod. Phys. Lett. A11 (1996) 675.

[16] T. Asaka and Ken-ichi Hikasa, Phys. Lett. B345 (1995) 36.

[17] R. Godbole, B. Mukhopadhyaya and M. Nowakowski, Phys. Lett. B352 (1995) 388.

[18] D. Ghosh, R. Godbole and B. Mukhopadhyaya, Phys. Rev. D55, (1997) 3150.

[19] OPAL Collaboration, K. Ackerstaff et al., CERN-EP/98-093, (1998) (hepex/9808014).

[20] A.G. Akeroyd, IFIC/98/48 (1998) (hep-ph/9806337).

[21] L3 Collaboration, M. Acciarri et al., Phys. Lett. B431 (1998) 437.

[22] ALEPH Collaboration, R. Barate et al., Phys. Lett. B412 (1997) 155.

[23] H. Haber, G. Kane and T. Sterling, Nucl. Phys. B161 (1979) 493; M.D. Diaz and T.J. Weiler, preprint VAND-TH-94-1 (1994) (hep-ph/9401259); A.Stange, W. Marciano and S. Willenbrock, Phys. Rev. D49 (1994) 1354; A.G. Akeroyd, Phys. Lett. B368 (1996) 89; A.G. Akeroyd, J. Phys. G24 (1998) 1983.

[24] A. Turcot, private communication.

[25] Cristal Ball Collaboration, D. Antreasyan et al., Phys. Lett. B251 (1990) 204.

[26] OPAL Collaboration, K. Ackerstaff et al., Eur. Phys. J. C5 (1998) 19.

[27] Delphi Collaboration, P. Abreu et al,. Phys. Lett. B420 (1998) 140. 
[28] A. Djouadi, J. Kalinowski and P.M. Zerwas, Z. Phys. C70 (1996) 435.

[29] I wish to thank A. Arhrib for pointing out that the formula displayed in Ref. 28 for $\Gamma\left(H^{ \pm} \rightarrow W^{*} A(h)\right)$ requires a extra multiplicative factor of 0.5 .

[30] S. Chakrabarti, D. Choudhury, R.M. Godbole and B. Mukhopadhyaya, Phys. Lett. B434 (1998) 347.

[31] J.F. Gunion, Int. J. Mod. Phys. A13 (1998) 2277.

[32] S. Komamiya et al., Phys. Rev. D38 (1988) 2158.

[33] K. Cheung, R.J.N. Phillips and A. Pilaftsis, Phys. Rev. D51 (1995) 4731. 